\title{
SECONDARY PREVENTION AFTER ACUTE CORONARY SYNDROME IN PATIENTS WITH TYPE 2 DIABETES: RESULTS FROM A ROUTINE CLINICAL PRACTICE
}

M. Cayón ${ }^{1}$ A. Del Río², C. García-Figueras ${ }^{3}$, P. Rubio ${ }^{3}$

Endocrinology and Nutrition Unit ${ }^{1}$. Cardiolology Unit ${ }^{2}$. Internal Medicine Unit ${ }^{3}$.

Hospital S.A.S. Jerez de la Frontera, Jerez de la Fra., Spain.

\section{INTRODUCTION}

Secondary prevention of coronary artery disease (CAD) in patients with type 2 diabetes mellitus (T2DM) has shown benefit but the level of control is still disappointing. Most of the results are documented within clinical trials. There isn't enough information in routine clinical practice at short-term tracing. The main aim of this study is to collect information about the level of control of major risk factors in non-selected patients.

\section{METHODS/DESIGN}

Observational and retrospective study. Patients with T2D who had suffered myocardial infarction between January and December 2012 were included.

Data provided by clinical history, with special regard to major cardiovascular risk factors were recorded. Optimal control of risk factors was defined by current clinical guidelines. Clinical events and achievement of targets at baseline, 6 months and 1-year after coronary event were recorded.

\section{RESULTS}

Data from forty-eight T2DM patients (47.9\% females, mean age: 70.6 \pm 8.7 years, mean diabetes duration: $10.9 \pm 9$ years) were collected. $79.2 \%$ had hypertension and $66.7 \%$ had hyperlipidemia (table 1 ).

Patients with higher level of HbA1c were more likely to receive drugeluting stent for coronary revascularazation (table 2 ).

At baseline, proportion of patients who met target for non-smoking, SBP, LDL-C and HbA1c was $89.6 \%, 62.2 \%, 40 \%$ and $22.2 \%$ respectively.

The rates of patients with adequate control at 6 and 12 months after discharge are summarized in table 3.

Although follow-up HbA1c level was lower than at baseline, no significantly difference was observed $(7.9 \pm 1.3 \%$ vs $7.3 \pm 1.2 \%$ vs $7.3 \pm 2.1 \% ; p=0.06$ ).

\begin{tabular}{|lc|}
\hline \multicolumn{2}{|l|}{ Table 1: Epidemiological variables $(\mathbf{n}=\mathbf{4 8})$} \\
\hline Gender Female / Male (\%) & 47.9 / 52.1 \\
\hline Age (years) & $70.6 \pm 8.7$ \\
\hline T2DM duration (years) & $10.9 \pm 9$ \\
\hline Hypertension (\%) & 79.2 \\
\hline Hyperlipidemia (\%) & 66.7 \\
\hline Current smokers (\%) & 10.4 \\
\hline Multivessel coronary disease (\%) & 57.5 \\
\hline ACS without ST-segment elevation (\%) & 70.8 \\
\hline
\end{tabular}

\begin{tabular}{|l|c|c|c|}
\hline $\begin{array}{l}\text { Table 2: Differences according to stent used } \\
\text { for coronary revascularization }\end{array}$ \\
\hline Stent & $65.9 \pm 8.6$ & $73.6 \pm 7.6$ & 0.003 \\
\hline Age (years) & $8.5 \pm 1.4$ & $7.6 \pm 1.2$ & 0.04 \\
\hline \begin{tabular}{l} 
HbA1c (\%) \\
\hline $\begin{array}{c}\text { Single-vessel coronary } \\
\text { disease (\%) }\end{array}$
\end{tabular} & 38.9 & 13.8 & 0.04 \\
\hline $\begin{array}{c}\text { Optimal distal } \\
\text { coronary bed (\%) }\end{array}$ & 50 & 10.3 & 0.003 \\
\hline
\end{tabular}

\begin{tabular}{|c|c|c|c|c|}
\hline Cardiovascular risk factor on target & Baseline & 6 months follow-up & 12 months follow-up & $\mathrm{p}$ \\
\hline Tobacco use cessation & $89.6 \%$ & $93.9 \%$ & $97.1 \%$ & 0.01 \\
\hline Systolic blood pressure & $62.2 \%$ & $74.4 \%$ & $71.4 \%$ & 0.004 \\
\hline LDLc & $40 \%$ & $70 \%$ & $83.3 \%$ & $<0.001$ \\
\hline HbA1c & $22.2 \%$ & $41,4 \%$ & $35.5 \%$ & NS \\
\hline \multicolumn{5}{|l|}{ Serum level (median; SD) } \\
\hline $\operatorname{LDLc}(\mathrm{mg} / \mathrm{dl})$ & $116.2 \pm 41.6$ & $42.1 \pm 6.8$ & $75.5 \pm 26.2$ & 0.013 \\
\hline HbA1c (\%) & $7.9 \pm 1.3$ & $7.3 \pm 1.2$ & $7.3 \pm 2.1$ & NS \\
\hline
\end{tabular}

NS: not significant

\section{CONCLUSIONS}

- Our outcomes from a routine clinical practice are relatively poor and don't differ much from the trials results.

- The rate of adequate control for each risk factor was similar at 6 months and at 1-year after coronary event. It reflects that there is an early improvement that is maintained after optimizing treatment. 